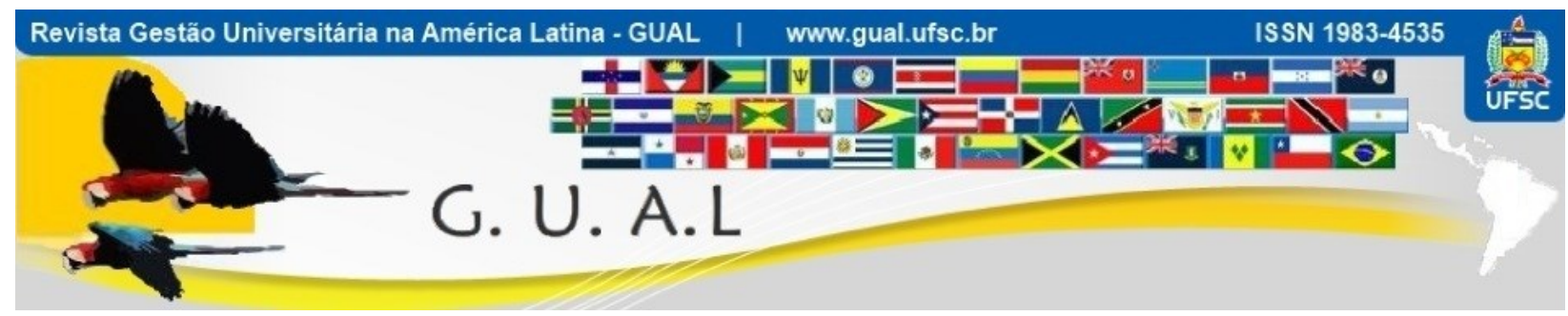

DOI: http://dx.doi.org/10.5007/1983-4535.2017v10n4p90

\title{
O EFEITO MODERADOR DA MOTIVAÇÃO INTERNA E EXTERNA E DO CONTROLE VOLITIVO NA EXPERIÊNCIA DE CONSUMO: UMA ANÁLISE SOB A ÓTICA DO ENSINO A DISTÂNCIA
}

\section{MOTIVATION, VOLITIVE CONTROL AND CONSUMPTION EXPERIENCE: AN ANALYSIS FROM THE DISTANCE LEARNING PERSPECTIVE}

\author{
Renata de Sousa da Silva Tolentino, Doutoranda \\ Universidade FUMEC \\ rsstolentino@globo.com \\ Cid Gonçalves Filho, Doutor \\ Universidade FUMEC \\ cid@,fumec.br
}

Cristiana Fernandes de Muylder, Doutora Universidade FUMEC cristiana.muylder@fumec.br

Plínio Rafael Reis Monteiro, Doutor Universidade Federal de Minas Gerais - UFMG preisufmg@,gmail.com

Ricardo José Vaz Tolentino, Mestre Universidade FUMEC vaztolentino@gmail.com

Recebido em 25/novembro/2016

Aprovado em 16/outubro/2017

Sistema de Avaliação: Double Blind Review

Esta obra está sob uma Licença Creative Commons Atribuição-Uso. 


\title{
RESUMO
}

A formação acadêmica dos cursos de administração e ciências contábeis prevê que o egresso O modelo contemporâneo da Educação a Distância $(\mathrm{EaD})$ contempla métodos, bases e padrões em desenvolvimento cujos critérios de qualidade ainda estão sendo estabelecidos e testados. Ademais, os motivos que levam os alunos a se matricularem em curso à distância são diversificados, sendo por vezes motivados por fatores extrínsecos (questões de tempo, distância física e condições financeiras) ou intrínsecos (afinidade, curiosidade e valorização do auto aprendizado). Este estudo buscou comparar se as motivações dos discentes influenciam o processo de avaliação da experiência com a modalidade de EaD. O estudo contemplou a elaboração de um modelo de avaliação da qualidade de ensino e dos serviços e com variáveis mediadoras (satisfação e imagem) e consequências comportamentais (orgulho, arrependimento e lealdade). Foi feito um levantamento com uma amostra de alunos de uma instituição que recentemente adotou o $\mathrm{EaD}$ como parte complementar as disciplinas presenciais. A amostra foi dividida de acordo com a maior motivação intrínseca ou extrínseca em relação a disciplina de EaD e foram testados modelos estruturais pelo método de PLS, onde se aplicou o teste de Smith-Satterthwait para verificar diferenças entre os modelos estruturais dos grupos, bem como das suas médias. Os resultados mostraram que alunos motivados internamente (com controle sobre seu comportamento) e externamente (sem controle sobre seu comportamento) avaliam e compreendem a experiência de ensino de forma distinta, sendo tais constatações o ponto de partida para fomentar o debate que considerou as implicações teóricas, práticas, limitações e caminhos para investigações futuras.

Palavras-chave: Marketing de Relacionamento. Ensino a Distância (EaD). Controle Volitivo.

\begin{abstract}
Contemporary distance learning approach comprises methods, standards and patterns in development in which quality standards are still been established and tested. Furthermore, it's factual the diversity of students motivations when they choose distance learning methods, since some could be internally (timing issues, logistics and financial conditions) or externally (positive attitude, curiosity and self-taught liking) motivated. This study seeks to identify the moderating role of internal and external motivation in the student experience with distance learning. The article proposes a theoretical model that addresses the relation between learning and services quality, relationship mediators (satisfaction and image) and behavioral consequences (proud, regret and loyalty). Was conducted a survey with undergraduate students of a high education institution that recently adopt distance learning as a complementary tool to conventional classes. The sample was split in two accordingly with the degree of internal or external motivation of the students towards distance learning. Structural Equation Models (SEM) using Partial Least Squares (PLS) estimation, were tested within and standardized weights for each sub-sample were statistically compared using the SmithSatterthwait test. Results shown that internally motivated students (that exhibit volitive control) evaluate and view the learning experience distinctly. These facts were the starting point to stimulate the debate around implications for theory, practice, limitations and directions for future studies.
\end{abstract}

Keywords: Relationship Marketing.Distance Learning. Volitive Control. 


\section{INTRODUÇÃO}

A educação a distância acompanha a evolução das tecnologias da informação e da comunicação (TICs). Alunos e professores podem ensinar e aprender em diferentes locais e autônoma organizando o tempo e atividades.

O Ensino a Distância $(\mathrm{EaD})$ faz parte do processo educacional contemporâneo (MAIA e MATTAR, 2007).

$\mathrm{O}$ crescimento sem precedentes da educação superior à distância aliada a critérios de qualidade recentes (Instituto Nacional de Estudos e Pesquisas Educacionais Anísio Teixeira [INEP], 2008) mostram a importância da definição de critérios e métodos de avaliação destes cursos. Em especial, deve-se ter atenção destacada às necessidades e expectativas dos discentes que buscam esta modalidade de ensino e mercado (Alonso, 2010).

Os motivos que levam os alunos a se matricularem em curso à distância são diversificados. A maior cobertura geográfica e maior flexibilidade de horários propiciada pela modalidade tornam o EaD uma prática caracterizada pela afiliação, abrangência e democracia (Nunes, 1994). Neste quesito, promove-se a inclusão de pessoas que, por condições externas e alheias à sua vontade, tem no EaD uma alternativa viável de formação. Por tal razão, muitos indivíduos por questões de tempo, distância física e condições financeiras podem optar por realizar cursos à distância (NISKIER, 2000).

A literatura destaca, entretanto, que esta não é a motivação única para que um aluno curse disciplinas a distância. Por vezes, faltam ao aluno condições favoráveis para que ele realize a disciplina ou um curso de forma presencial. Na literatura acadêmica este tema é tratado pelo que se convenciona de controle volitivo, fator limitante das escolhas no contexto de consumo em geral. Estudos sob como a limitação de recursos delimita os comportamentos são bastante difundidos na área de marketing (AJZEN, 1985; WIECHETEK, 2011). De forma geral se pode argumentar que, na impossibilidade de se satisfazer através de produtos/serviços "ideais", os consumidores podem buscar alternativas que supram parcialmente suas necessidades. Em um contexto de $\mathrm{EaD}$, pode-se dizer que alguns indivíduos podem ser "forçados" a se matricular em cursos de EaD por, em determinado momento, não perceberem nos cursos presenciais alternativas viáveis de formação. Notadamente, indivíduos que escolhem um curso de EaD por interesse e desejo interno podem ter expectativas diferentes daqueles que acreditam que só têm esta alternativa viável. 
Neste contexto busca-se compreender: Quais as diferenças entre a avaliação da experiência no EaD de alunos que escolheram a modalidade voluntariamente daqueles que não viram alternativas viáveis de ensino? Buscou-se comparar, portanto, a experiência de ensino de alunos de EaD motivados externamente (por falta de alternativas) e internamente (por interesse, desejo ou curiosidade).

Entende-se que o tema é relevante diante da busca por compreensão da escolha ou perfil do aluno que opta por estudo na modalidade à distância como proposto por FARIAS, PEREIRA, OLIVEIRA e GALDINO (2014) e avançar nas reflexões que indicam específicos estudantes. Para este estudo foi realizado estudo descritivo de natureza qualitativa e quantitativa propondo modelo de comparação da escolha do estudante. Além desta introdução o artigo está composto em outros três capítulos sendo o segundo referente à revisão de literatura. $\mathrm{O}$ terceiro sobre a metodologia utilizada seguido dos resultados e discussões. $\mathrm{O}$ quarto tratou das considerações finais seguido das referências e apêndices.

\section{REVISÃO DE LITERATURA}

A revisão da literatura deste artigo contemplou os tópicos Educação à distância, Qualidade e satisfação de serviços, Lealdade e Motivação interna e externa e controle volitivo.

\subsection{EDUCAÇÃO À DISTÂNCIA: PANORAMA HISTÓRICO, EVOLUÇÃO E CARACTERÍSTICAS}

A revolução tecnológica propiciada pela profusão do computador e popularização da internet gerou uma transformação drástica nos meios de produção (CASTELLS, 1999), gerando níveis de difusão do conhecimento inimagináveis antes do advento da microeletrônica (RAVETZ, 1996). Como parte integrante do sistema social, os métodos de ensino foram profundamente afetados pela onipresença do ciberespaço, sendo neste contexto os lócus da educação a distância que se popularizou com a difusão da internet e adoção da prática por entidades de ensino renomadas de caráter pública e privada (CASAGRANDE, KLERING e KRUEL, 2008).

As características mais marcantes do $\mathrm{EaD}$ são a separação física entre professor e aluno, a utilização de meios eletrônicos de comunicação e o uso da comunicação de mãodupla (tutorias, orientações, observações sobre trabalhos realizados, esclarecimento de dúvidas e avaliações finais) (NUNES, 1994). O pressuposto desta abordagem é a busca do 
conhecimento pelo discente a despeito da ausência do professor (Cardoso \& Pestana, 2001). A $\mathrm{EaD}$ e a internet ainda expandem as barreiras da sala de aula possibilitando explorar conteúdos e disciplinas para fronteiras que transcendem os limites da sala de aula tradicional (MAGDALENA e MESSA, 1998; PALLOFF e PRATT, 2002).

\subsection{QUALIDADE E SATISFAÇÃO COM OS SERVIÇOS}

Segundo Zeithaml (1988) um serviço é de qualidade somente quando iguala ou supera as expectativas do cliente. A mensuração da qualidade proposta por Zeithaml, Parasuraman e Berry (1985) é um dos métodos mais populares para conceber a avaliação de serviços. Este modelo, denominado de Servqual, parte da premissa de que a qualidade do serviço é obtida comparando-se o serviço esperado com o recebido pelo cliente. Neste cenário as expectativas emergem com uma importância destacada, sendo influenciada por fatores como a comunicação verbal, experiências pessoais e comunicações externas.

Enquanto a qualidade refere a percepção da performance geral do serviço em si, a satisfação representa uma consequência da compra e uso de uma oferta, resultante da comparação feita pelos consumidores das recompensas e custos da transação em relação às consequências antecipadas (CHURCHILL e SUPRENANT, 1982). Assim a satisfação mede o grau em que os resultados para o cliente foram afetivamente positivos (Harvey, 1998). Operacionalmente, a satisfação é similar à atitude, podendo ser entendida como a soma das satisfações com vários atributos do bem ou serviço (CHURCHILL e SUPRENANT, 1982). Após estas definições iniciais percebe-se estudos que concentram análise de qualidade de serviços educacionais como Saeta, Popadiuk, e Teixeira (2003), Granito (2008) e Ferreira (2016).

\subsection{LEALDADE}

Existem várias definições para o que se chama de fidelização ou "lealdade do cliente". Griffin (2001) diz que um cliente fiel é aquele, dentre outras coisas, repete suas compras com regularidade, faz a comunicação positiva de produtos / serviços a outras pessoas, resiste à pressão da concorrência e tolera eventuais problemas na prestação de serviços sem desertar.

Sob um ponto de vista afetivo a lealdade pode ser entendida como a vontade de um cliente de permanecer adquirindo a oferta de uma empresa ao longo do tempo, comprando e utilizando bens e serviços com base repetida e preferencial (LOVELOCK e WRIGHT, 2001). 
É importante destacar que a lealdade é um construto que vários autores têm analisado como consequência da satisfação (ZEITHAML e BITNER, 2003). Para Oliver (1999), satisfação e lealdade são construtos distintos, mas intimamente relacionados. Enquanto a satisfação é o início da transição afetiva a lealdade propriamente dita é um resultado final. Ainda segundo o autor, diversas formas de lealdade (ou fases) são distinguíveis, quais sejam: a afetiva, cognitiva, conativa e a ação de compra. Além desta visão mais abrangente ainda pode-se avançar como proposto por Pereira, Cavalheiro, Potrich, Campara e Paraboni (2016) quanto a lealdade e opção dos discentes.

\subsection{MOTIVAÇÃO INTERNA-EXTERNA E CONTROLE VOLITIVO}

Os processos decisórios de compra são influenciados por fatores externos e internos que afetam como o consumidor pensa, avalia e age (ENGEL et al., 1995). Sob uma perspectiva de tomada de decisão racional aspectos cognitivos são tidos como fundamentais na influência do consumidor (MOWEN e MINOR, 2003). Dentre as variáveis categorizadas como diferenças individuais que moldam a decisão de consumo, destaca-se a motivação como fator crucial nas ações de compra.

$\mathrm{O}$ entendimento dos fatores motivacionais remonta aos estudos clássicos sobre necessidades humanas, tendo em Maslow uma de suas figuras mais proeminentes. A hierarquia das necessidades, apesar de ter sofrido contestações ao longo dos anos, ainda é uma sólida estrutura de análise do consumidor. De forma geral, pode-se atribuir a esta hierarquia duas fontes básicas de motivações. A motivação externa refere-se usualmente às necessidades básicas (fisiológicas e de segurança) que são determinadas por condições alheias à vontade e preferência individual (exemplo: falta de comida, sentimento de insegurança, busca por melhores perspectivas futuras). Já a motivação interna ocorre nos níveis elevados da pirâmide, onde o indivíduo busca satisfazer desejos pessoais, como a auto realização e a superação pessoal.

A distinção entre motivações internas e externas adotada neste estudo se coaduna com a perspectiva do controle volitivo. Para Fishbein e Ajzen (1975) o controle volitivo representa a capacidade de executar comportamentos desejados. Segundo Ajzen (1985) quando fatores externos impedem ou dificultam que o indivíduo realize os comportamentos por ele almejados, ele se torna menos propenso a empreender atividades que visem o alcance destas metas. Por isto, mesmo que o indivíduo deseje realizar um comportamento, a falta de controle 
volitivo pode impedi-lo. Neste caso, estratégias alternativas podem emergir com o intuito de suprir as necessidades do consumidor. Neste estudo, levantaram-se as principais motivações internas e externas que levaram os indivíduos a se matricularem em cursos ou disciplinas de EaD. Na instituição pesquisada os fatores externos foram a falta de tempo, ausência de vagas no curso ou na disciplina presencial e a necessidade de adiantar o curso (pela reprovação ou abandono anterior do curso presencial).

\section{MODELO DE PESQUISA}

Observando-se a revisão da literatura definiu-se o modelo de pesquisa com o objetivo de verificar empiricamente relações entre as variáveis de interesse. Este modelo foi concebido por intermédio da conjugação de revisão bibliográfica, pesquisa qualitativa ampla e avaliação de validade de conteúdo ampla (contando com entrevistas e grupos de foco com alunos, professores, coordenadores, técnicos-administrativos e diretores).

A base deste modelo é a divisão da qualidade em dois componentes ligados à aspectos operacionais e administrativos dos serviços: Qualidade de ensino (sistema de ensino de excelência, integrado à prática profisssional à um custo/preço justo) e Qualidade dos serviços (estrutura de apoio ao ensino que permite o desenvolvimento das atividades pedagógicas e oferece um espaço agradável de convivência).

A Qualidade de Ensino foi dividida na Qualidade dos Professores, Qualidade da Estrutura de EaD, Qualidade das Disciplinas à Distância, Qualidade da Coordenação e Qualidade do Curso. Já a Qualidade dos Serviços foi composta pela avaliação da seção de ensino, tesouraria, biblioteca, estrutura e laboratórios. Outras variáveis que compõem o modelo são a Imagem (modelo ECSI), satisfação (Servqual) e lealdade (inspirada em OLIVER, 1981). A lealdade foi medida na forma de propensão a se matricular em outros cursos/módulos de $\mathrm{EaD}$ (Lealdade de ação), arrependimento (usada como Proxy da chance de abandono do curso - Lealdade cognitiva) e o orgulho (Lealdade afetiva).

Adicionalmente inseriu-se a auto avaliação dos alunos, como um reflexo e formador das expectativas, presentes tanto nos modelos de lealdade, satisfação e qualidade previamente definidos. A suposição é que alunos mais esforçados sejam mais exigentes em relação à prestação de serviços. A figura em sequência mostra o modelo de pesquisa concebido e testado de forma comparativa entre alunos motivados interna e externamente. 
Figura 1 Modelo hipotético de pesquisa.

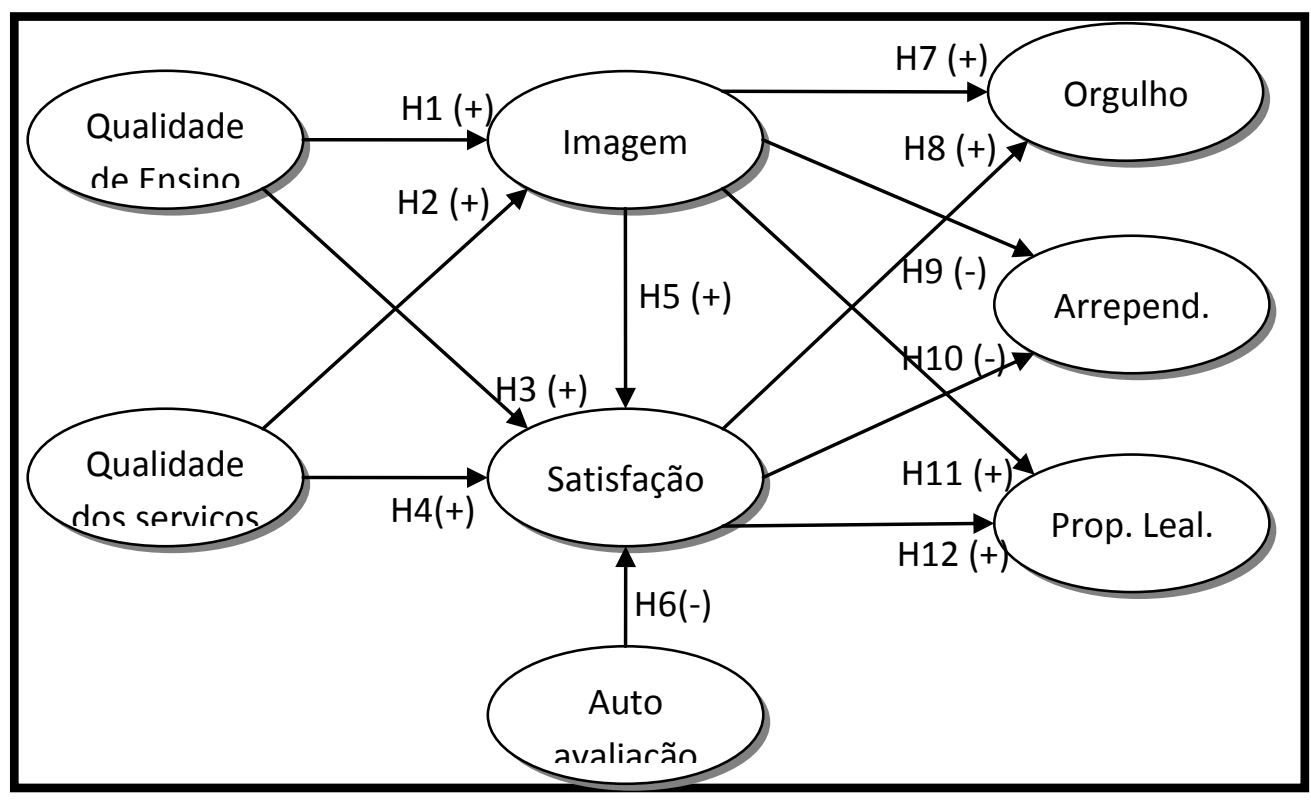

Fonte: Elaborado pelos autores.

\section{METODOLOGIA}

\subsection{AMOSTRA}

Este estudo contou com uma amostra dos alunos de graduação tradicional e de tecnologia de uma instituição que recentemente adotou a perspectiva de ensino à distância. $\mathrm{O}$ levantamento contou com 113 participantes de um total de 200 alunos (56,5\% de taxa de resposta) aptos a responder a pesquisa por terem passado pela experiência recente e experimental do $\mathrm{EaD}$ na instituição. Salienta-se que as respostas foram obtidas de alunos que estavam cursando somente algumas disciplinas a distância e não o curso completo. Após os procedimentos de análise exploratória de dados, 92 casos válidos foram obtidos, o que em nível gerencial, permite considerar as conclusões sobre os dados na instituição com intervalo de confiança acima de $95 \%$ e erro abaixo amostral de $6 \%$.

\subsection{FASE EXPLORATÓRIA}

As escalas foram elaboradas por pesquisadores especialistas em metodologia de pesquisa e psicometria (doutores e mestres). As perguntas foram definidas com a ajuda de dezenas de entrevistas qualitativas e grupos de foco com diferentes públicos alvo da instituição (alunos, professores, coordenadores, técnicos administrativos e direção). Os resultados foram apurados com métodos tradicionais de avaliação de confiabilidade e validade 
e melhorias foram conduzidas através de aplicações periódicas dos instrumentos a toda população de alunos da IES. A base do questionário tem sido usado a vários anos para avaliar a qualidade e satisfação de alunos da instituição pesquisada. Empregou-se uma escala do tipo Likert com 11 pontos visando um melhor tratamento das mesmas como contínuas (NUNNALLY e BERNSTEIN, 1994). O detalhamento dos itens de operacionalização dos construtos é apresentado adiante na etapa de validação do instrumento.

\subsection{VERIFICAÇÕES PRELIMINARES DAS MEDIÇÕES}

$\mathrm{Na}$ análise de dados ausentes foi necessário excluir 21 respostas com mais de 27 dados ausentes e as questões 33 (Tem excelentes laboratórios específicos do curso), 62 (Salas de aula adequadas) e 73 (Recomendo às pessoas a estudarem na IES X). Após solucionar tais pontos poucos dados ausentes dispersos aleatoriamente $(p>0,05)$ entre variáveis e casos foram repostos por meio do procedimento EM.

Os dados se desviaram significativamente da normalidade levando a preferência pelo método de estimação de mínimos quadrados parciais (PLS). Outliers identificados pela distância interquartílica foram substituídos. Os outliers multivariados (valor $\mathrm{p}<0,01$ na distância de Mahalanobis) praticamente não geraram alterações nos resultados, sendo, portanto, mantidos na análise.

Pressupostos de multicolinearidade (pelo valor VIF ajustado ao tamanho da amostra e número de variáveis), linearidade (30 diagramas de dispersão escolhidos aleatoriamente) e variâncias relativas (nenhuma razão das variâncias superou o valor de 10 pontos) não demonstraram violações (KLINE, 1998). Portanto, estas etapas revelam condições propicias para testar os modelos.

\subsection{ANÁLISE DA FIDEDIGNIDADE DAS MEDIÇÕES}

Buscou-se fazer uma avaliação subjetiva e sistemática das escalas, garantindo a validade de conteúdo das perguntas. A dimensionalidade foi avaliada pela análise fatorial, conforme sugerem Netemeyer, Sharma e Bearden (2003) (eigenvalues maiores que um e rotação oblimin direta). À exceção da avaliação geral da disciplina de EaD (questões de 16 a 24) e avaliação do coordenador, os demais quesitos avaliados foram encontrados com somente uma dimensão. 
A validade convergente foi analisada pelos valores das cargas fatoriais superiores a 0,63, indicando comunalidades superiores a 0,40 (mais de 40\% de confiabilidade) (BOLLEN, 1989). Os casos que não tingiram este critério de validade foram excluídos na etapa de validação dos modelos subsequentes.

As escalas apresentaram elevada consistência interna com valores de Alpha de Cronbach acima de 0,8 em sua grande maioria. Foi realizada a análise da confiabilidade composta e variância média extraída, conforme sugerem Cair Jr., Tacham e Black (1988). A confiabilidade composta dos construtos ficou de 0,7 e a variância média extraída (AVE), o que indica elevada confiabilidade e consistência das medições. A validade discriminante analisada pelo esquema de Farnel e Parker (1981) foi alcançada, pois a variância média extraída é sempre superior a variância compartilhada entre os construtos (R2).

\subsection{TESTE DO EFEITO MODERADOR DA MOTIVAÇÃO INTERNA E EXTERNA}

A comparação e teste do modelo foi feito usando a técnica de equações simultâneas com variáveis latentes (NETEMEYER, SHARMA e BEARDEN, 2003, p.76). Como os desvios da normalidade foram elevados e a amostra pequena empregou-se a estimação de múltiplos estágios denominada de Mínimos Quadrados Parciais (Partia Letas Suares - PLS) (HAENLEIN e KAPLAN, 2004, p.283). Chin (1998) sugere que o tamanho da amostra mínimo a ser aplicado em um estudo com PLS seja de 10 a 5 vezes o bloco de indicadores do construto com maior número de indicadores ou o construto que tem maior número de variáveis independentes.

Para ajustar o modelo a esta possibilidade e permitir a representação de fatores de segunda ordem (qualidade do ensino e dos serviços), cada dimensão encontrada na etapa de validação foi usada como um indicador latente no modelo estrutural. Portanto, exceção feita aos construtos qualidade de ensino e dos serviços, todos os demais construtos foram representados por indicadores únicos, isto, trata-se um modelo muito similar a análise de caminhos (KLINE, 1998). Sob tais condições a amostra em cada grupo é grande o suficiente para a estimação.

Visando verificar diferenças entre os indivíduos quem elevada motivação interna ou externa para realização da disciplina ou cursos à distância, procedeu-se ao teste de dois modelos estruturais um para cada amostra de estudantes. Para identificar os grupos com maior tendência à Motivação Externa para cursas disciplinas de EaD, dividiu-se a amostra pela 
mediana das perguntas válidas da dimensão Motivação Externa $(0,00)$ resultando em um grupo de Motivação Externa com n=30 e um grupo de motivação interna com $n=62$. Pode-se entender o grupo de alunos motivados externamente como aqueles que não tinham controle sobre sua decisão sendo "obrigados" a cursar a disciplina a distância, enquanto os motivados internamente tinham outras opções, mas escolhera a modalidade EaD. Em sequência ajustaram-se modelos via PLS em ambos os grupos. Os resultados são mostrados nos modelos em sequência.

Figura 2 Modelo hipotético de pesquisa: grupo com motivação externa.

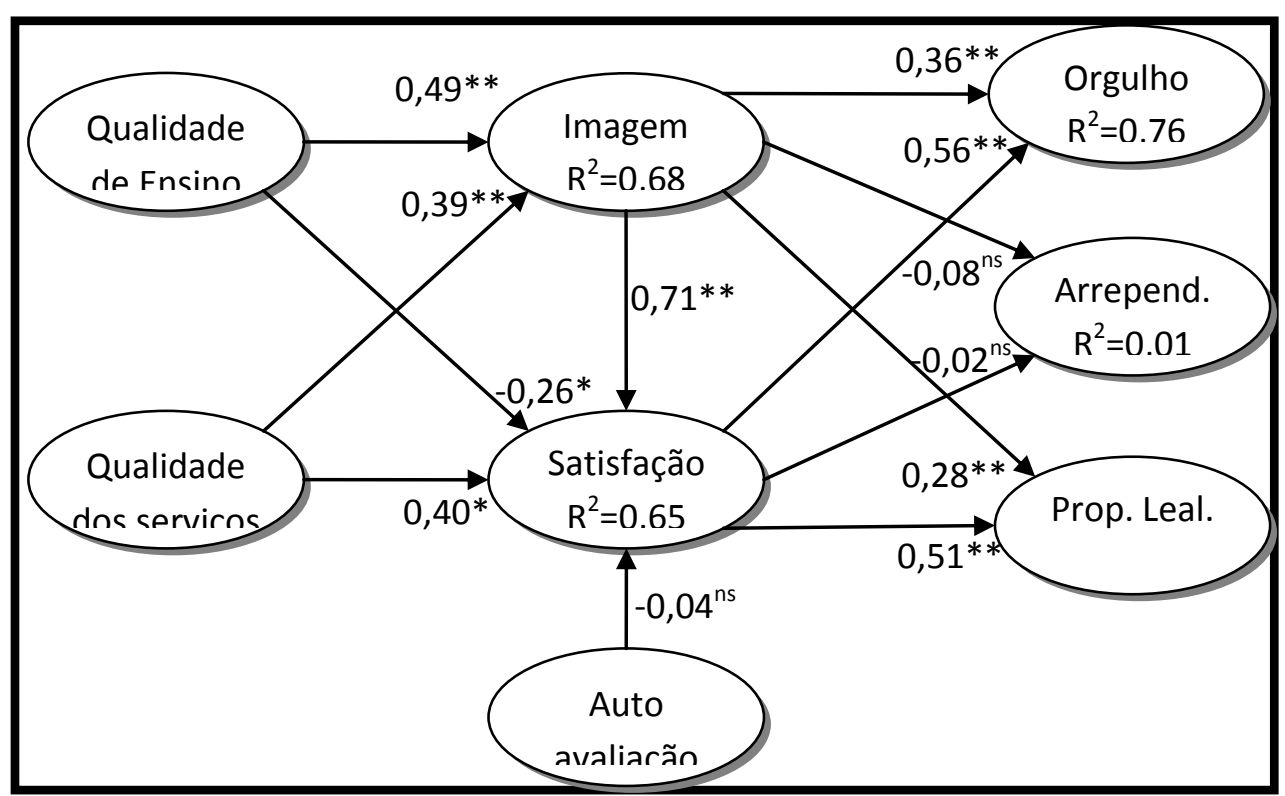

Fonte: Dados da pesquisa.

Figura 3 Modelo hipotético de pesquisa: grupo com motivação interna.

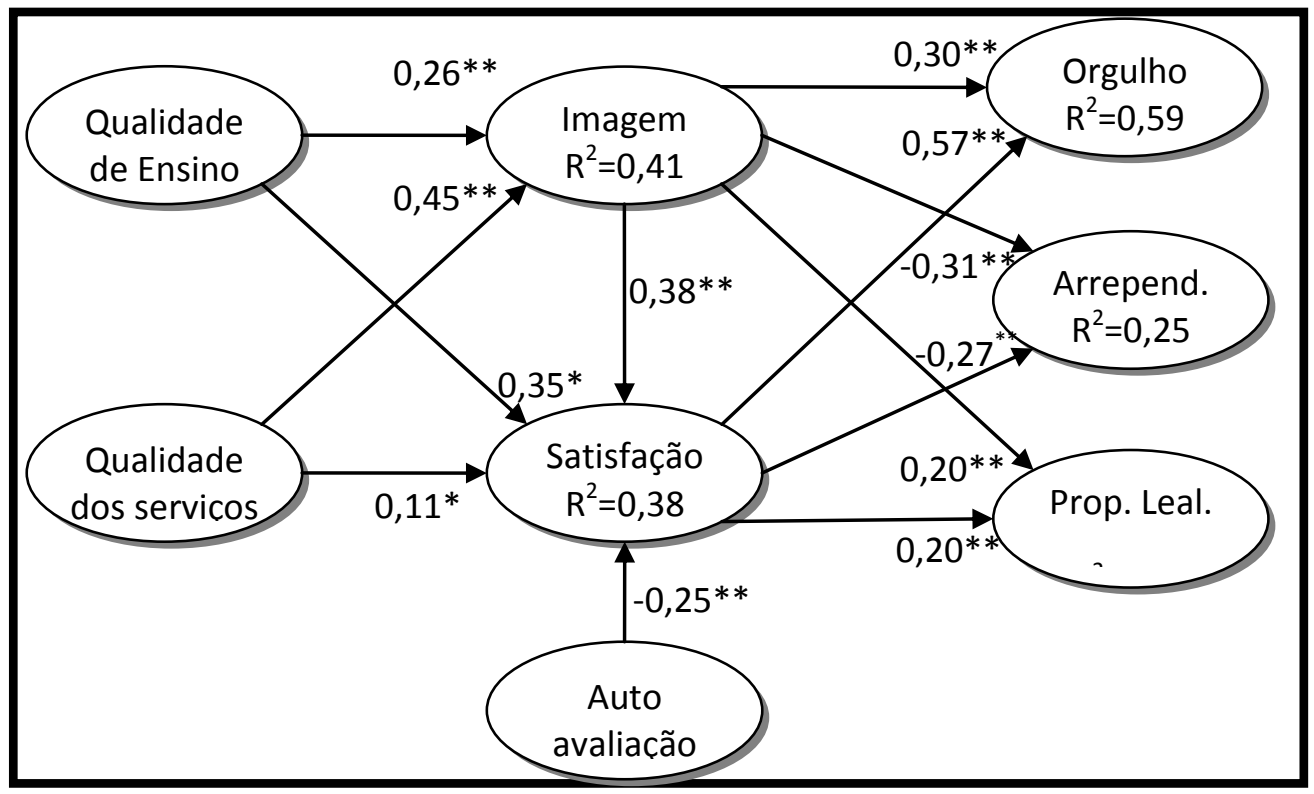

Fonte: Dados da pesquisa. 


\section{O EFEITO MODERADOR DA MOTIVACÃ̃O INTERNA E EXTERNA E DO CONTROLE VOLITIVO NA EXPERIÊNCIA DE CONSUMO: UMA ANÁLISE SOB A ÓTICA DO ENSINO A DISTÂNCIA DOI: http://dx.doi.org/10.5007/1983-4535.2017v10n4p90}

Observações: Os valores apresentados junto às setas indicam o valor das cargas padronizadas. NS indica uma carga não significativa $(\mathrm{P}>0,05)$. ** indica a significância da carga ao nível de $1 \%(\mathrm{p}<0,01)$; * indica a significância da carga ao nível de $5 \%(\mathrm{p}<0,05)$. O Valor R2 indica o percentual de variância explicada dos construtos endógenos.

Nas Figuras 2 e 3 notam-se grandes diferenças entre os modelos que analisam o efeito das variáveis dentre os grupos pesquisados. Destaca-se que os efeitos, de forma geral, parecem ser mais fortes dentre o grupo que é motivado externamente, pois os valores de R2 tendem a ser maiores (inclusive caso apliquemos o ajuste pelo tamanho da amostra e número de variáveis preditoras). Igualmente, os pesos padronizados divergem bastante entre grupos. Aplicou-se o teste de Smith-Satterthwait, uma adaptação do teste t que é descrito por Chin (2000), para identificar diferenças entre os grupos. Os resultados completos podem ser vistos na Tabela 1.

Tabela 1 Pesos padronizados por grupo e testes de significância.

\begin{tabular}{|c|c|c|c|c|c|c|c|c|c|c|}
\hline \multirow[b]{2}{*}{ RELAÇÕES } & \multicolumn{4}{|c|}{ MOTIVAÇÃO EXTERNA } & \multicolumn{3}{|c|}{ MOTIVAÇÃO INTERNA } & \multicolumn{3}{|c|}{ COMPARAÇÃO } \\
\hline & PESO & ERRO & $\mathbf{T}$ & SIG. & PESO ERRO & $\mathbf{T}$ & SIG. & DIF & $\mathbf{T}$ & SIG. \\
\hline $\begin{array}{l}\text { Auto avaliação } \Rightarrow \\
\text { Satisfação }\end{array}$ & $-0,045$ & 0,075 & 0,603 & 0,552 & $-0,249 \quad 0,065$ & 3,806 & 0,000 & 0,204 & 2,135 & 0,035 \\
\hline Imagem $\Rightarrow$ Satisfação & 0,714 & 0,095 & 7,478 & 0,000 & $0,376 \quad 0,096$ & 3,911 & 0,000 & 0,338 & 2,662 & 0,009 \\
\hline $\begin{array}{l}\text { Qualidade de ensino } \\
\Rightarrow \text { Imagem }\end{array}$ & 0,488 & 0,058 & 8,435 & 0,000 & $0,256 \quad 0,074$ & 3,453 & 0,001 & 0,232 & 5,738 & 0,000 \\
\hline $\begin{array}{l}\text { Qualidade de ensino } \\
\Rightarrow \text { Satisfação }\end{array}$ & $-0,265$ & 0,081 & 3,260 & 0,003 & $0,354 \quad 0,083$ & 4,270 & 0,000 & $-0,619$ & $\begin{array}{c}- \\
5,703\end{array}$ & 0,000 \\
\hline $\begin{array}{l}\text { Qualidade dos } \\
\text { Serviços } \Rightarrow \text { Satisfação }\end{array}$ & 0,396 & 0,076 & 5,211 & 0,000 & $0,107 \quad 0,109$ & 0,987 & 0,328 & 0,289 & 2,468 & 0,015 \\
\hline $\begin{array}{l}\text { Qualidade dos } \\
\text { Serviços } \Rightarrow \text { Imagem }\end{array}$ & $\mathbf{0 , 3 9 3}$ & $\mathbf{0 , 0 7 0}$ & 5,598 & $\mathbf{0 , 0 0 0}$ & $\mathbf{0 , 4 4 9} \quad \mathbf{0 , 0 7 7}$ & 5,868 & $\mathbf{0 , 0 0 0}$ & $-0,057$ & $\overline{1,228}$ & 0,223 \\
\hline $\begin{array}{l}\text { Imagem } \\
\Rightarrow \text { Arrependimento }\end{array}$ & $-0,082$ & 0,109 & 0,748 & 0,460 & $-0,308 \quad 0,099$ & 3,113 & 0,003 & 0,226 & 3,365 & 0,001 \\
\hline $\begin{array}{l}\text { Satisfação } \Rightarrow \\
\text { Arrependimento }\end{array}$ & $-0,020$ & 0,103 & 0,195 & 0,847 & $-0,269 \quad 0,093$ & 2,905 & 0,005 & 0,249 & 3,922 & 0,000 \\
\hline Imagem $\Rightarrow$ Orgulho & 0,364 & 0,075 & 4,866 & 0,000 & $0,3020,074$ & 4,088 & $\mathbf{0 , 0 0 0}$ & 0,062 & 1,311 & 0,193 \\
\hline $\begin{array}{l}\text { Satisfação } \Rightarrow \\
\text { Orgulho }\end{array}$ & $\mathbf{0 , 5 5 9}$ & 0,091 & 6,143 & $\mathbf{0 , 0 0 0}$ & $\mathbf{0 , 5 6 6} \quad \mathbf{0 , 0 7 3}$ & 7,777 & $\mathbf{0 , 0 0 0}$ & $-0,006$ & $\overline{-}, 119$ & 0,905 \\
\hline $\begin{array}{l}\text { Satisfação } \Rightarrow \\
\text { Propensão à 1EaDade }\end{array}$ & 0,509 & 0,084 & 6,070 & 0,000 & $0,198 \quad 0,056$ & 3,515 & 0,001 & 0,310 & 6,423 & 0,000 \\
\hline $\begin{array}{l}\text { Imagem } \Rightarrow \\
\text { Propensão à lealdade }\end{array}$ & 0,280 & $\mathbf{0 , 0 8 0}$ & 3,519 & 0,001 & $\mathbf{0 , 1 9 7} \quad \mathbf{0 , 0 7 0}$ & 2,820 & 0,006 & 0,083 & 1,696 & 0,093 \\
\hline
\end{tabular}

Fonte: dados da pesquisa. OBS: Peso é o valor do peso padronizado; ERRO é o erro padrão da estimativa; T é o valor T de teste; SIG. É a significância do teste; DIF. é a diferença entre os pesos dos grupos.

A Tabela 2, precedente mostra os pesos significativos no estudo, bem como as diferenças (coluna comparação). Mostrasse que grande parte das diferenças entre os pesos 
mostraram-se significativas entre os grupos $(p<0,05)$. Considerando que os grupos têm tanto médias como variabilidades diferentes, pode-se atribuir as diferenças nos pesos a diferenças efetivas no efeito das variáveis. Além das diferenças nos pesos outra diferença notável foi a influência das dimensões de qualidade em cada um dos quesitos avaliados.

Tabela 2 Cargas fatoriais dos quesitos avaliados sobre as dimensões de qualidade.

\begin{tabular}{lc|c|c|}
\hline \hline QUESITO AVALIADO & DIMENSÃO & EXTERNA & INTERNA \\
\hline Professores & Qualidade de ensino & $0,787(5)$ & $0,463(6)$ \\
Comunicação & Qualidade de ensino & $0,884(2)$ & $0,798(2)$ \\
$\begin{array}{l}\text { Facilidade } \\
\text { Acompanhamento do }\end{array}$ & Qualidade de ensino & $0,887(1)$ & $0,677(4)$ \\
coordenador & & & \\
Atendimento coordenador & Qualidade de ensino & $0,128(7)$ & $0,676(5)$ \\
Curso & Qualidade de ensino & $0,139(6)$ & $0,397(7)$ \\
Disciplinas & Qualidade de ensino & $0,874(3)$ & $0,818(1)$ \\
Laboratórios & Qualidade de ensino & $0,845(4)$ & $0,755(3)$ \\
Secretaria & Qualidade dos Serviços & $0,879(2)$ & $0,695(5)$ \\
Tesouraria & Qualidade dos Serviços & $0,740(4)$ & $0,774(4)$ \\
Biblioteca & Qualidade dos Serviços & $0,769(3)$ & $0,839(1)$ \\
Estrutura & Qualidade dos Serviços & $0,740(5)$ & $0,829(2)$ \\
\hline
\end{tabular}

Fonte: dados da pesquisa. OBS: os valores dentro dos parênteses (ao lado das cargas fatoriais) mostra a ordem de grandeza dos pesos dentro de cada grupo e dimensão de qualidade.

\section{CONSIDERAÇÕES FINAIS}

O teste do modelo mostrou que alunos com motivações internas para fazer uma disciplina de $\mathrm{EaD}$ são mais exigentes quanto a qualidade de ensino do que alunos que fazem a disciplina por falta de opções (motivação externa). Dentre alunos que são obrigados a fazer módulos a distância, o peso total da qualidade de ensino sobre a satisfação e praticamente nulo $(0,49 \times 0,71-0,26=0,09)$ enquanto no grupo internamente motivado e relativamente elevado $(0,26 \times 0,38+0,35=0,45)$. Isto indica que aqueles alunos que fazem uma disciplina ou modulo de EaD por impossibilidade de fazer o curso presencial tem expectativas mais baixas em relação ao ensino quando comparados aos alunos que buscam uma disciplina ou curso a distância. Também pode revelar certo pré-conceito de alunos acostumados ao curso presencial (motivados externamente) quanto a qualidade dos cursos a distância, fazendo com suas expectativas sejam menores.

Coaduna com esta conclusão o fato do auto avaliação não exercer impacto significativo sobre a satisfação no caso de alunos motivados externamente. Neste caso, a explicação e que sendo menores as expectativas de alunos obrigados a fazer disciplinas de 
$\mathrm{EaD}$, seu esforço, naturalmente mais baixo que dos alunos internamente motivados, não influencia o quanto eles ficam satisfeitos com a instituição. Conforme se nota, dentre alunos internamente motivados, aqueles com maiores níveis de esforços, tendem a menor satisfação, já que presumivelmente tem maiores expectativas.

Quanto a qualidade da estrutura a situação é inversa, pois alunos que fazem o curso motivados externamente percebem a estrutura como mais relevante $(0,39 \times 0,71+0,40=0,68)$ que os alunos motivados internamente $(0,45 \times 0,38+0,11=0,28)$. Isto revela que alunos que gostariam de fazer a disciplina ou curso em modalidade EaD mostram-se menos exigentes quanto à estrutura da instituição, pois sabem que seu contato com a estrutura ocorrera em poucas e pré-definidas ocasiões. Por outro lado, os alunos acostumados com a estrutura presencial tradicional transferem grande parte de suas expectativas com os serviços para os aspectos tangíveis da instituição, demonstrando que aspectos mais tangíveis e relacionados a organização física se tornam deveras relevantes estes alunos.

Interessa notar que a satisfação média de alunos interna e externamente motivados e exatamente igual $(9,00)$. Assim, apesar de terem diferentes expectativas, a instituição obtém o mesmo grau de atendimento das expectativas dos alunos.

Quanto as formas de lealdade mostraram-se diferenças significativas entre os grupos. O arrependimento não é afetado pela imagem e satisfação no grupo motivado externamente, enquanto no grupo motivado internamente este efeito e negativo e relativamente forte $(\square 0,30)$. Este resultado e congruente com as motivações destes grupos. Como o grupo motivado externamente não teve escolha sobre sua decisão, eles têm menos razoes para se arrepender caso o serviço não alcance níveis esperados de desempenho.

Em oposição, aqueles que escolheram ativamente o curso a distância, estariam mais propensos a se arrepender desta decisão. Sendo o arrependimento uma proxy da probabilidade abandono, é de se esperar que a evasão da disciplina seja mais provável dentre alunos motivados internamente (que queriam um curso de $\mathrm{EaD}$ ) do que dentre aqueles motivados externamente (que não tinham outra opção de curso no momento).

Quanto a propensão a se matricular em outros cursos de EaD no futuro, observa-se um maior efeito da imagem e, especialmente, satisfação no grupo motivado externamente. Isto demonstra claramente que quando confrontados com uma qualidade de ensino que supera suas expectativas os alunos que anteriormente tinham baixas expectativas e possivelmente algum pré-conceito contra o $\mathrm{EaD}$ se tornam mais propensos a se matricular em disciplinas ou 
cursos desta modalidade. Por outro lado, aqueles motivados internamente, por possivelmente já estarem satisfazendo suas necessidades educacionais com o curso de EaD, tem menos intenção de se matricular em outros cursos no futuro. De fato, a análise das medias de lealdade dos grupos, revela que indivíduos internamente motivados têm menor intenção $(7,29)$ que os externamente motivados $(7,53)$ de se matricular em outros cursos de EaD no futuro.

Finalmente o impacto da imagem e satisfação sobre o orgulho e estatisticamente equivalente, demonstrando que, em termos da lealdade em seu formativo mais afetivo (gostar ou não), alunos internamente e externamente motivados são afetados de forma similar (a média do grupo externamente motivado $=8,93$ e do grupo internamente motivados $=8,94$ ).

Finalmente aponta-se diversas diferenças entre a importância das dimensões de qualidade dentre os grupos. Pode-se dizer que dentre os alunos motivados externamente a qualidade de ensino apresenta os fatores com seguinte ordem de importância: 1) Facilidade de uso; 2) Comunicação eficaz; 3) Curso; 4) Disciplinas; 5) Professores; 6) Atendimento coordenador; 7) Acompanhamento do coordenador. Para alunos motivados internamente a seguinte ordem de relevância é apresentada: 1) Curso; 2) Comunicação eficaz; 3) Disciplinas; 4) Facilidade de uso; 5) Acompanhamento do coordenador; 6) Professores; 7) Atendimento coordenador.

Para a qualidade dos serviços mostrasse que alunos internamente motivados percebem maior importância no laboratório quando comparado ao grupo motivado internamente. Por outro lado, a Tesouraria e a Biblioteca, tendem a maior relevância dentre os alunos internamente motivados. De forma geral a ordem de importância dentre alunos internamente motivados é: 1) Estrutura; 2) Laboratórios; 3) Tesouraria; 4) Secretaria; 5) Biblioteca. Para alunos externamente motivados a ordem de importância é: 1) Tesouraria; 2) Biblioteca; 3) Estrutura; 4) Secretaria; 5) Laboratórios.

O estudo contribui a teoria e prática ao propor o desenvolvimento de um conjunto de pontos a serem priorizados na elaboração de programas de EaD. Em primeiro lugar destaca-se que para instituições e cursos em que o EaD entrara como recurso adicional, deve-se manter um bom vínculo com aspectos tangíveis dos serviços prestados. Neste caso os serviços de tesouraria e biblioteca são muito importantes para satisfazer os alunos, que possivelmente terão mais interesse em buscar conhecimentos nas fontes tradicionais (livros, revistas, artigos e periódicos). Por outro lado, os investimentos em laboratórios de informática são menos 
importantes que para alunos motivados internamente. Nestes casos também a ferramenta de apoio ao EaD deve enfatizar sua facilidade de uso sobre sua qualidade técnica e layout.

Já nos cursos que são "integralmente" a distância importa-se ter disciplinas, módulos, professores e uma ferramenta de $\mathrm{EaD}$ com uma comunicação e conexão inequívoca e disciplinas com acabamento supremo. Deve-se incentivar o uso de exemplos práticos, ilustrações, apresentação gráfica atraente, atividades de fixação e avaliações congruentes com o conteúdo ministrado. Neste caso os laboratórios de informática devem ser priorizados, apesar os recursos com biblioteca serem menos importantes.

$\mathrm{O}$ estudo lança um olhar crítico sobre a avaliação da qualidade em $\mathrm{EaD}$ e destaca o importante papel das necessidades dos alunos neste contexto. O modelo de pesquisa sugere que alunos com diferentes motivações tendem a expectativas distintas quanto aos serviços. Sugere-se que as proposições oriundas das diferenças estabelecidas entre os modelos de pesquisa sejam reavaliadas em contextos e públicos amplos a fim de suportar ou refutá-las como generalizações. Finalmente, quanto às limitações, destaca-se a ausência de múltiplos indicadores para as dimensões dos construtos finais do modelo, o uso de uma amostra pequena para teste de um modelo estrutural completo e o uso de amostragem não probabilísticas.

\section{REFERÊNCIAS}

AJZEN, I. . From intentions to actions: A theory of planned behavior. In Kuhl J. e Beckman J. (Eds.), Action-control: From cognition to behavior. pp. 11-39. Heidelberg, Germany: Springer. 1985. Recuperado em 10 Novembro de 2009 de http://www.people.umass.edu/aizen/publications.html.

ALONSO, K. M. A expansão do ensino superior no Brasil e a EaD: dinâmicas e lugares. Educação e Sociedade, 2010 31(113), 1319-1335.

BOLLEN, K. A. Structural equations with latent variables. 1989. New York: Wiley.

CARDOSO, F. C. e PESTANA, T. M. P. (2001) Treinamento online (e-learning). In BOOG, G. G. (Coord.). Manual de treinamento e desenvolvimento. São Paulo: Makron, 2001. pp. 205-220.

CASAGRANDE, L., KLERING, L. R. e KRUEL, A. J. (2008). Estudo comparativo de percepções de alunos de especialização latu sensu nas modalidades presencial e EaD. Anais do Encontro Anual da ANPAD, Rio de Janeiro, RJ, Brasil, 2008. 15.

CASTELLS, M.. A sociedade em rede. São Paulo: Paz e Terra.1999 
CHIN, W. W. Issues and Opinion on Structure Equation Modeling. MIS Quarterly, 1998, vol. 22, vii-xvi.

CHIN, W. W. Frequently Asked Questions - Partial Least Squares e PLS-Graph. 2000. Recuperado em 18 de março de 2010 de http://disc-nt.cba.uh.edu/chin/plsfaq.htm.

CHURCHILL, G. A., e SUPRENANT, C. An investigation into the determinants of customer satisfaction. Journal of Marketing Research, 1982. v. 19, p. 491-504, nov.

EADKLINE, R. B. Principles and practice of structural equation modeling. New York: The Guilford Press. 1998.

EADMOWEN, J. C., e MINOR, M. S. Comportamento do consumidor. São Paulo: Prentice Hall, 2003.

ENGEL, J. F., BLACKWELL, R. D., e MINIARD, P.W. Comportamento do Consumidor. Rio de Janeiro: Livros Técnicos e Científicos, 1995.

FARIAS, A., PEREIRA, M., OLIVEIRA, S., e GALDINO, S. Os Cursos Técnicos da Educação a Distância (EAD): uma Análise dos Perfs dos Alunos Ingressos do Nordeste Brasileiro. In Anais do Workshop de Informática na Escola, 2014. (Vol. 20, No. 1, p. 400).

FERREIRA, J. B. Avaliação da qualidade de serviços em uma instituição privada de ensino superior do Alto Paranaíba/MG, 2016.

FISHBEIN, M., e AJZEN, I. Belief, Attitude, Intention, and Behavior: An Introduction to Theory and Research. REaDing, MA: Addison-Wesley. 1975.

FORNELL, C., e LARCKER, D. F. Evaluating structural equation models with unobservablevariables and measurement error. Journal of Marketing Research, 1981, vol. 18 , pp. 39-50, February.

GRANITO, R. A. N. Educação a distância e estilos de aprendizagem: elaboração de um protocolo de qualidade para ambientes virtuais de ensino (Doctoral dissertation, Universidade de São Paulo). 2008.

GRIFFIN, J. Um programa de fidelização. HSM Management, Boston, 2001, Vol. 5, N. 28 , set.-out.

HAENLEIN, M., e KAPLAN, A.M. A beginner's guide to partial least squares (PLS) analysis, Understanding statistics, 2004, vol. 3, N. 4, pp. 283-297.

HAIR JR, J. F., R. E. ANDERSON, R. L. TATHAM, e W. C. BLACK. Multivariate data analysis. 5th ed. Upper Saddle River: Prentice Hall, 1988.

HARVEY, J. Service quality: a tutorial. Journal of Operations Management, Amsterdam, 1998. vol. 16, n. 5, Oct. pp. 583-897. 
Instituto Nacional de Estudos e Pesquisas Educacionais Anísio Teixeira [INEP]. (2007).

Educação a Distância cresce mais ainda entre os cursos superiores. Recuperado em 19 de dezembro de 2009 em

$<$ http://www.inep.gov.br/imprensa/noticias/censo/superior/news07_01.htm>.

International School for Social and Business Studies, Celje, Slovenia.

LOVELOCK, C., e WRIGHT, L. Serviços, marketing e gestão. São Paulo: Saraiva, 2001.

MAGDALENA, B. C., e MESSA, M. R. P. Educação à distância e Internet em sala de aula. Revista Brasileira de Informática na Educação, 1998, 2(1), 25-34.

MAIA, C., e MATTAR, J. ABC da EaD: a educação a distância hoje. São Paulo: Pearson Prentice Hall, 2007;

NETEMEYER, R. G. BEARDEN, W. O., e SHARMA, S. Scaling procedures: Issues and Applications. SAGE, 2003.

NISKIER, A. Educação à distância: a tecnologia da esperança. $2^{\mathrm{a}}$ ed. São Paulo: Loyola. 2000 .

NUNES, I.B. Noções de Educação a Distância. Revista Educação a Distância n. 4/5, Dez., Brasília, Instituto Nacional de Educação a Distância, 1994. pp. 7-25

NUNNALY, J. C. e BERNSTEIN, I. H. Psychometric Theory. 3 ed. New York; McGrawHill, 1994.

OLIVER, R. L. Measurement and evaluation of satisfaction processes in retail settings. Journal of Retailing, 1991. Vol. 57, pp. 25-48.

OLIVER, R. L. Whence Consumer Loyalty. Journal of Marketing, 1999. Vol. 63 Special Issue, pp. 33-44.

PALLOFF, R. M., e PRATT, K. Construindo comunidades de aprendizagem no ciberespaço. Porto Alegre: Artmed, 2002.

PEREIRA, B. A. D., CAVAlHEIRO, E. A., POTRICH, A. C. G., CAMPARA, J. P., e PARABONI, A. L. . Avaliação Da Satisfação E Lealdade Discente: Validação de Ferramenta em Universidades Brasileiras Usando o Modelo European Customer Satisfaction Index (ECSI). Perspectivas Contemporâneas, 2016. 11(1), 100-119.

RAVETZ, J. R. The microcybernetic revolution and the dialectics of ignorance. In: ZIAUDDIN, S. e RAVETZ, J. R. (ed.). Cyberfutures: cultures and politics on the information superhighway. London: Pluto Press. 1996. pp. 42-60.

SAETA, B. R. P., POPADIUK, S., e TEIXEIRA, M. L. M. Avaliação de serviços educacionais no ensino superior: o ponto de vista de alunos regulares e vestibulandos portadores de deficiência. Organizações e Sociedade, 2003. 10(27), 27-39. 
WIECHETEK, Lukasz. Using e-Learning tools to Support Cooperation between Science and Business: Case of Synergy Project, Knowledge as Business Opportunity:

Proceedings of the Management, Knowledge and Learning International Conference 2011.

ZEITHAML, V. A. e BITNER, M. J. Marketing de Serviços: A Empresa com Foco no Cliente. 2003. 2.ed. Porto Alegre: Bookman.

ZEITHAML, V. A. Consumer Perception of Price, Quality and Value : a means-end model and synthesis of evidence. Journal of Marketing, Chicago, 2008. Vol. 52, jul.

ZEITHAML, V. A., PARASURAMAN, A., e BERRY, L. L. Problems and strategies in services marketing. Journal of Marketing, 1985. vol. 49, Chicago, Spring. 